\title{
A New Edge Detection Method for CT-Scan Lung Images
}

\author{
${ }^{1}$ Ali El-Zaart and ${ }^{2}$ Toufic El-Arwadi \\ Department of Mathematics and Computer Science, Faculty of Science, \\ Beirut Arab University, Lebanon

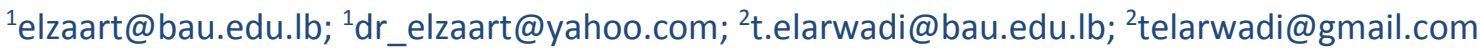

\begin{abstract}
Lung cancer is one of the most serious health problems in the world. Lung Computer-Aided Diagnosis (CAD) is a potential method to accomplish a range of quantitative tasks such as early cancer and disease detection, analysis of disease progression. Edge detection in Lung image is a fundamental step in CAD system and many algorithms have suggested for edge detection in image. In this paper a new operator has developed for edge detection based on finite difference method. Experimentation showed that the proposed operator gave good results in detection the edges in Lung images.
\end{abstract}

Keywords: Lung image; adaptive mask; finite difference method; gradient.

\section{Introduction}

L Computed tomography, more commonly known as a CT or CAT scan, is a diagnostic medical test that, like traditional x-rays, produces multiple images or pictures of the inside of the body. A chest CT Scan produces images of sufficient image quality to detect many lung diseases and abnormalities [].Because CT scans are able to detect even very small nodules in the lung, chest CT is especially effective for diagnosing lung cancer at its earliest, most curable stage [2-5]. Survival from lung cancer is directly related to its growth at its detection time. . The earlier the detection is, the higher the chances of successful treatment. Lung Computer-Aided Diagnosis (CAD) is a potential method to accomplish a range of quantitative tasks such as early cancer and disease detection, analysis of disease progression. Edge detection in Lung image is a fundamental step in CAD system and many algorithms have suggested for edge detection in image [1]. The purpose of this paper is to develop a new $5 \times 5$ operator for edge detection in Lung images. This operator is based on the finite difference method. The rest of this paper is organized as follows. Section 2 presents the related work of edge detection. Section 3 presents the proposed method. Experimental results are shown in section 4. Finally this paper presents conclusion and future work in section 5.

\section{The Existing Ranking Methods}

Medical image edge detection is an important work for object recognition of the human organs such as lungs, and it is an essential pre-processing step in medical image segmentation [1]. In some applications it may be useful to classify image pixels into anatomical regions, such as bones, muscles, and blood vessels, while in others into pathological regions, such as cancer, tissue deformities and multiple sclerosis lesions. The goal of the lung edge detection required for the computer aided diagnosis from CT scan images is to 
essentially separate the voxels corresponding to the lung cavity in the axial CT scan slices from the surrounding lung anatomy. Over the years, many methods have been proposed for detecting edges in images. Some of the earlier methods, such as the Sobel and Prewitt detectors used local gradient operators $[1,7,8]$ to obtain spatial filter masks. The procedure is to compute the sum of products of the mask coefficients with the intensity values in the region encompassed by the mask [10]. In addition, the Canny edge detector which depends on the Gaussian distribution for obtaining the operators for the gradient and Laplacian masks is a well-known edge detector [10]. In this paper, we propose $5 \times 5$ masks for edge detection in Lung CT images. The method is based on the 1D and 2D Taylor's. In our previous work, the developed a $5 \times 5$ operator based on the 1D and 3D Taylor's and we obtain an operator composed of two parameters $\alpha$ and $\beta$, in this paper we added a third parameter on the operator in order to have more general operator than the one in [6]. In next section, we will explain the new operator.

\section{The Modified Operators}

In this section, we will improve the operator developed in our previous work [6]. The previous operator is based on 2D Taylor expansion and we used two parameters $\alpha$ and $\beta$ in order to construct the operators (masks). In this paper we will modify the previous operator by adding a new parameter $\gamma$. In the follows we will explain the modified operator. First in section 3.1, we will explain the 1D Taylor's formula and then in section 3.2 we will explain the 2D Taylor's expansion.

In pure mathematics, given a function $f \in \mathrm{C}^{\mathrm{n}+1}$ that is, all its derivatives $\mathrm{f}^{(\mathrm{j})}, \mathrm{j}=1, \ldots, \mathrm{n}+1$ are continuous. Let $\mathrm{x}$ be a fixed point, then for $\mathrm{h}$ close to 0 , there exists $t \in(0,1)$ such that:

$$
f(x+h)=f(x)+\sum_{i=1}^{n} \frac{h^{i}}{i !} f^{(i)}(x)+f^{(n+1)}(c) \frac{h^{n+1}}{(n+1) !}
$$

Where $\mathrm{c}=\mathrm{x}+$ th. The term $\mathrm{R}_{\mathrm{n}}:=\mathrm{f}^{(\mathrm{n}+1)}(\mathrm{c}) \frac{\mathrm{h}^{\mathrm{n}+1}}{(\mathrm{n}+1) !}$ is called the remainder term. Using the big -0 notation, we abbreviate the formula (1) as follow:

$$
f(x+h)=f(x)+\sum_{i=1}^{n} \frac{h^{i}}{i !} f^{(i)}(x)+O\left(h^{n+1}\right)
$$

Let $\mathrm{X}$ be a normed vector space, If $\mathrm{f}: \mathrm{X} \rightarrow \mathrm{R}$ is differentiable $\mathrm{n}+1$ times on $\mathrm{X}$, it may be expanded by Taylor's formula, for each $\mathrm{x} \in \mathrm{X}$ :

$$
f(x+h)=f(x)+D f(x) \cdot h+\frac{1}{2 !} D^{2} f(x) \cdot h^{2}+\cdots+\frac{1}{n !} D^{n} f(x) \cdot h^{n}+R_{n}(x)
$$

Where $R_{n}(x)=\frac{1}{(n+1) !} D^{n+1} f(c) \cdot h^{n+1}$. The k-Frechet Derivative of $f$ at $x$ is being denoted by $D^{k} f(x)$, to be viewed as a multilinear map from $X^{k}$ to $R$. The $\cdot h^{k}$ notation means to evaluate $D^{k} f(x)$ at $(h, \ldots, h)$.

If $X=R^{2}, D^{k}$ has the following expression $\frac{1}{k !} D^{k} f(x) \cdot h^{k}=\sum_{|J|=k} \frac{1}{J !} \frac{\partial^{|J|}}{\partial x^{J}}(x) h^{J}$

Where $\mathrm{J}$ is a vector of 2 components. The multi-index $\mathrm{J}$ runs through all combinations such that $\mathrm{J}_{1}+\mathrm{J}_{2}=$ $|\mathrm{J}|=\mathrm{k}$ in the sum and $\mathrm{J} !=\mathrm{J}_{1} ! \mathrm{J}_{2}$ ! 
Ali El-Zaart and Toufic El-Arwadi; A New Edge Detection Method for CT-Scan Lung Images. Journal of Biomedical Engineering and Medical Imaging, Volume 2, No 5, October (2015) , pp 1-9

From equation (3), we can rewrite the 2D Taylor expansion as follows:

$$
f(x+l, y+k)=f(x, y)+l \frac{\partial f}{\partial x}+k \frac{\partial f}{\partial y}+\frac{1}{2}\left(\frac{\partial^{2} f}{\partial x^{2}} l^{2}+2 h l \frac{\partial^{2} f}{\partial x \partial y}+\frac{\partial^{2} f}{\partial y^{2}} k^{2}\right)+0\left(l^{3}+k^{3}\right)
$$

From this formula, we take the values of $\mathrm{l}=(-1)^{\mathrm{n}} \mathrm{h}$ and $\mathrm{k}=(-1)^{\mathrm{m}} \mathrm{h}$, for $\mathrm{n}= \pm 1$ and $\mathrm{m}= \pm 1$ to obtain the following 4 formulas :

-For $\mathrm{l}=\mathrm{h}$ and $\mathrm{k}=\mathrm{h}$, we obtain:

$$
f(x+h, y+h)=f(x, y)+h \frac{\partial f}{\partial x}+h \frac{\partial f}{\partial y}+\frac{h^{2}}{2}\left(\frac{\partial^{2} f}{\partial x^{2}}+2 \frac{\partial^{2} f}{\partial x \partial y}+\frac{\partial^{2} f}{\partial y^{2}}\right)+O\left(h^{3}\right)
$$

- For $\mathrm{l}=-\mathrm{h}$ and $\mathrm{k}=\mathrm{h}$, we obtain:

$$
f(x-h, y+h)=f(x, y)-h \frac{\partial f}{\partial x}+h \frac{\partial f}{\partial y}+\frac{h^{2}}{2}\left(\frac{\partial^{2} f}{\partial x^{2}}-2 \frac{\partial^{2} f}{\partial x \partial y}+\frac{\partial^{2} f}{\partial y^{2}}\right)+O\left(h^{3}\right)
$$

- For $\mathrm{l}=\mathrm{h}$ and $\mathrm{k}=-\mathrm{h}$, we obtain:

$$
f(x+h, y-h)=f(x, y)+h \frac{\partial f}{\partial x}-h \frac{\partial f}{\partial y}+\frac{h^{2}}{2}\left(\frac{\partial^{2} f}{\partial x^{2}}-2 \frac{\partial^{2} f}{\partial x \partial y}+\frac{\partial^{2} f}{\partial y^{2}}\right)+O\left(h^{3}\right)
$$

- For $\mathrm{l}=-\mathrm{h}$ and $\mathrm{k}=-\mathrm{h}$, we obtain:

$$
f(x-h, y-h)=f(x, y)-h \frac{\partial f}{\partial x}-h \frac{\partial f}{\partial y}+\frac{h^{2}}{2}\left(\frac{\partial^{2} f}{\partial x^{2}}+2 \frac{\partial^{2} f}{\partial x \partial y}+\frac{\partial^{2} f}{\partial y^{2}}\right)+O\left(h^{3}\right)
$$

By multiplying the equations (5) and (7) by $\alpha$ and the equation (6) and ( 8 ) by $\beta$, we obtain the following formula:

$$
\begin{aligned}
\alpha f(x+h, y+h) & +\alpha f(x+h, y-h)+\beta f(x-h, y+h)+\beta f(x-h, y-h) \\
= & (2 \alpha+2 \beta) f(x, y)+(2 \alpha-2 \beta) h \frac{\partial f}{\partial x}+(\alpha+\beta) h^{2} \Delta f+O\left(h^{3}\right)
\end{aligned}
$$

Where $\Delta f$ is the Laplacian of $f(x, y)$.

By replacing $h$ by $2 h$ in (9), we obtain:

$$
\begin{gathered}
\alpha f(x+2 h, y+2 h)+\alpha f(x+2 h, y-2 h)+\beta f(x-2 h, y+2 h)+\beta f(x-2 h, y-2 h) \\
=(2 \alpha+2 \beta) f(x, y)+(4 \alpha-4 \beta) h \frac{\partial f}{\partial x}+4(\alpha+\beta) h^{2} \Delta f+O\left(h^{3}\right)
\end{gathered}
$$

Now multiply (9) by $\gamma$ and add to (10) we deduce that:

$$
\begin{aligned}
f(x+2 h, y+2 h) & +\alpha f(x+2 h, y-2 h)+\beta f(x-2 h, y+2 h)+\beta f(x-2 h, y-2 h)+\gamma \alpha f(x+h, y+h) \\
+ & \gamma \alpha f(x+h, y-h)+\gamma \beta f(x-h, y+h)+\gamma \beta f(x-h, y-h) \\
= & (2 \alpha+2 \beta)(\gamma+1) f(x, y)+(2 \alpha-2 \beta) h(\gamma+2) \frac{\partial f}{\partial x}+(\alpha+\beta) h^{2}(\gamma+4) \Delta f+O\left(h^{3}\right)
\end{aligned}
$$

By taking $h$ close to zero, we can neglect $h^{2}$ and $h^{3}$ and we deduce the following approximation for $\frac{\partial f}{\partial x}$ 


$$
\begin{aligned}
& \frac{\partial f}{\partial x}=\frac{1}{(2 \alpha-2 \beta) h(\gamma+2)}(\alpha f(x+2 h, y+2 h)+\alpha f(x+2 h, y-2 h)+\beta f(x-2 h, y+2 h) \\
& +\beta f(x-2 h, y-2 h)+\gamma \alpha f(x+h, y+h)+\gamma \alpha f(x+h, y-h)+\gamma \beta f(x-h, y+h) \\
& +\gamma \beta f(x-h, y-h)-(2 \alpha+2 \beta)(\gamma+1) f(x, y))
\end{aligned}
$$

Note that the approximation (11) is of order 1 , if $\gamma=-4$, the term $(\alpha+\beta) h^{2}(\gamma+4) \Delta$ fisappears and the approximation is of order 2 .

By multiplying the equations (5) and (6) by $\alpha$ and the equation (7) and (8) by $\beta$, we can obtain the following formula:

$$
\begin{array}{r}
\alpha f(x+h, y+h)+\alpha f(x-h, y+h)+\beta f(x+h, y-h)+\beta f(x-h, y-h) \\
=(2 \alpha+2 \beta) f(x, y)+(2 \alpha-2 \beta) h \frac{\partial f}{\partial y}+(\alpha+\beta) h^{2} \Delta f+O\left(h^{3}\right)
\end{array}
$$

Replace $\mathrm{h}$ by $2 \mathrm{~h}$ in $(12)$ to obtain

$$
\begin{aligned}
& \alpha f(x+2 h, y+2 h)+\alpha f(x-2 h, y+2 h)+\beta f(x+2 h, y-2 h)+\beta f(x-2 h, y-2 h) \\
= & (2 \alpha+2 \beta) f(x, y)+(4 \alpha-4 \beta) h \frac{\partial f}{\partial y}+4(\alpha+\beta) h^{2} \Delta f+O\left(h^{3}\right)
\end{aligned}
$$

In Paper [6], the order of the approximation is 1, to increase the order of the approximation we introduce a new parameter $\gamma$. We will show that for a special value of $\gamma$ the order of the approximation is 2 . Now multiply (12) by $\gamma$ and add to (10) we deduce :

$$
\begin{aligned}
\alpha f(x+2 h, y+2 h)+\alpha f(x-2 h, y+2 h)+\beta f(x+2 h, y-2 h)+\beta f(x-2 h, y-2 h)+\gamma \alpha f(x+h, y+h) \\
+\gamma \alpha f(x-h, y+h)+\gamma \beta f(x+h, y-h)+\gamma \beta f(x-h, y-h) \\
=(2 \alpha+2 \beta)(\gamma+1) f(x, y)+(2 \alpha-2 \beta) h(\gamma+2) \frac{\partial f}{\partial y}+(\alpha+\beta) h^{2}(\gamma+4) \Delta f+O\left(h^{3}\right)
\end{aligned}
$$

Similarly, we can deduce the following approximation for $\frac{\partial \mathrm{f}}{\partial \mathrm{y}}$

$$
\begin{aligned}
& \frac{\partial f}{\partial y}=\frac{1}{(2 \alpha-2 \beta) h(\gamma+2)}(\alpha f(x+2 h, y+2 h)+\alpha f(x-2 h, y+2 h)+\beta f(x+2 h, y-2 h) \\
& +\beta f(x-2 h, y-2 h)+\gamma \alpha f(x+h, y+h)+\gamma \alpha f(x-h, y+h)+\gamma \beta f(x+h, y-h) \\
& +\gamma \beta f(x-h, y-h)-(2 \alpha+2 \beta)(\gamma+1) f(x, y))
\end{aligned}
$$

Note that the approximation (14) is of order 1 , if $\gamma=-4$, the term $(\alpha+\beta) h^{2}(\gamma+4) \Delta$ f disappears and the approximation is of order 2.

From the above formulas (11) and (14), we construct the $5 \times 5$ horizontal masks as follows:

$$
\mathrm{Mx}=\frac{1}{(2 \alpha-2 \beta) \mathrm{h}(\gamma+2)}\left(\begin{array}{ccccc}
\beta & 0 & 0 & 0 & \beta \\
0 & \gamma \beta & 0 & \gamma \beta & 0 \\
0 & 0 & (2 \beta+2 \alpha)(\gamma+1) & 0 & 0 \\
0 & \gamma \alpha & 0 & \gamma \alpha & 0 \\
\alpha & 0 & 0 & 0 & \alpha
\end{array}\right)
$$

The Sobel $5 \times 5$ horizontal and vertical mask are [1] 
Ali El-Zaart and Toufic El-Arwadi; A New Edge Detection Method for CT-Scan Lung Images. Journal of Biomedical Engineering and Medical Imaging, Volume 2, No 5, October (2015) , pp 1-9

Similarly, we can write the My Mask as following:

$$
\operatorname{My}=\frac{1}{(2 \alpha-2 \beta) h(\gamma+2)}\left(\begin{array}{ccccc}
\alpha & 0 & 0 & 0 & \beta \\
0 & \gamma \alpha & 0 & \gamma \beta & 0 \\
0 & 0 & (2 \beta+2 \alpha)(\gamma+1) & 0 & 0 \\
0 & \gamma \alpha & 0 & \gamma \beta & 0 \\
\alpha & 0 & 0 & 0 & \beta
\end{array}\right)
$$

The Sobel $5 \times 5$ horizontal and vertical mask are [1]

$$
\mathrm{Mx}=\left(\begin{array}{ccccc}
-1 & -4 & -6 & -4 & -1 \\
-2 & -8 & -12 & -8 & -2 \\
0 & 0 & 0 & 0 & 0 \\
2 & 8 & 12 & 8 & 2 \\
1 & 4 & 6 & 4 & 1
\end{array}\right) \text { and } M y=\left(\begin{array}{ccccc}
1 & 2 & 0 & -2 & -1 \\
4 & 8 & 0 & -8 & -4 \\
6 & 12 & 0 & -12 & -6 \\
4 & 8 & 0 & -8 & -4 \\
1 & 2 & 0 & -2 & -1
\end{array}\right)
$$

\section{Experimental Results}

In this section, we applied our proposed operator on three lung CT-Scan images and we choose $\alpha=1, \beta=-$ 2 and $\gamma=1$.

- Image figure 1a presents the original CT-Scan lung image. Image in figure $1 \mathrm{~b}$ presents the result of new operator in $\mathrm{x}$-direction. Image in figure $1 \mathrm{c}$ presents the result of new operator in y-direction. Image in figure $1 \mathrm{~d}$ presents the result of Sobel operator in $\mathrm{x}$-direction. Image in figure 1e presents the result of Sobel operator in y-direction. Note that in this image, our proposed operator gave better results than Sobel operator.

- Image figure $2 \mathrm{a}$ presents the original CT-Scan lung image. Image in figure $2 \mathrm{~b}$ presents the result of new operator in $\mathrm{x}$-direction. Image in figure $2 \mathrm{c}$ presents the result of new operator in y-direction. Image in figure $2 \mathrm{~d}$ presents the result of Sobel operator in $\mathrm{x}$-direction. Image in figure 2e presents the result of Sobel operator in y-direction. Note that in this image, our proposed operator gave better results than Sobel operator.

- Image figure 3a presents the original CT-Scan lung image. Image in figure 3b presents the result of new operator in $x$-direction. Image in figure $3 c$ presents the result of new operator in $y$-direction. Image in figure $3 d$ presents the result of Sobel operator in $x$-direction. Image in figure 3e presents the result of Sobel operator in y-direction. Note that in this image, our proposed operator gave better results than Sobel operator. 


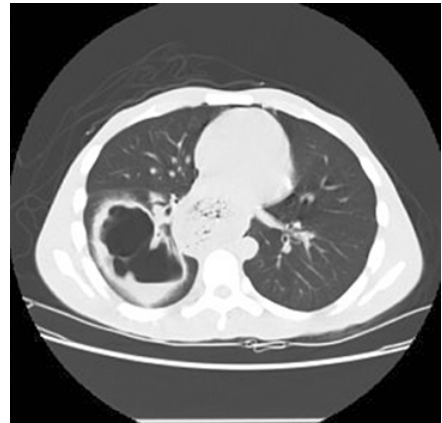

(a): Original CT-Lung image

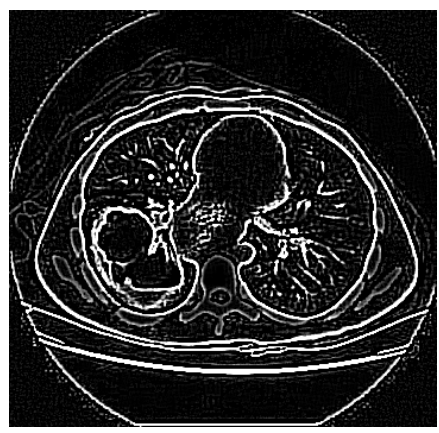

Result of the proposed $5 \times 5$ mask in y-direction

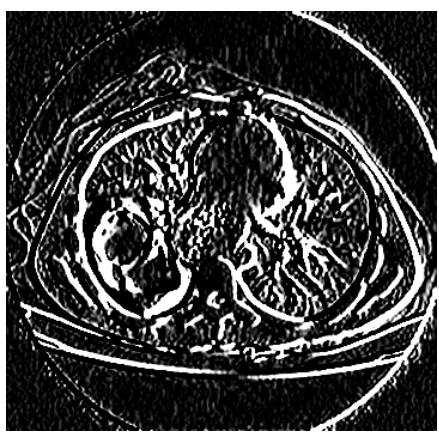

(e) : Sobel $5 \times 5$ Mask in y-direction

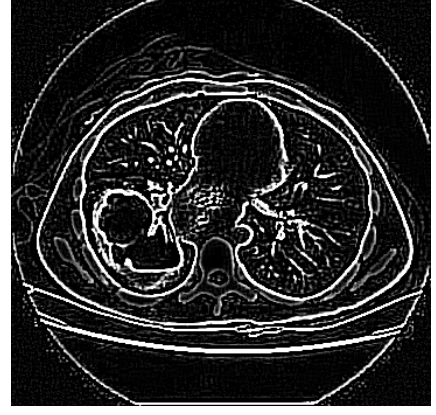

(b): Result of the proposed $5 \times 5$ mask in $x$-direction

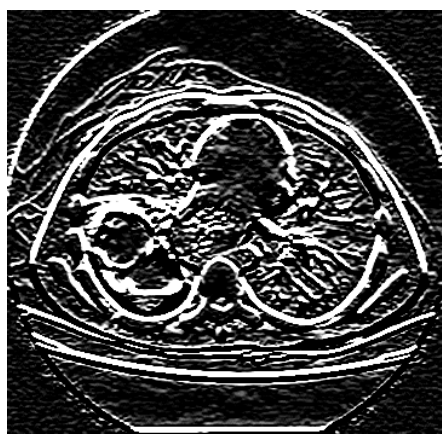

(d) Sobel $5 \times 5$ Mask in x-direction

Figure 1. Results of our proposed method and $5 \times 5$ Sobel masks 
Ali El-Zaart and Toufic El-Arwadi; A New Edge Detection Method for CT-Scan Lung Images. Journal of Biomedical Engineering and Medical Imaging, Volume 2, No 5, October (2015) , pp 1-9

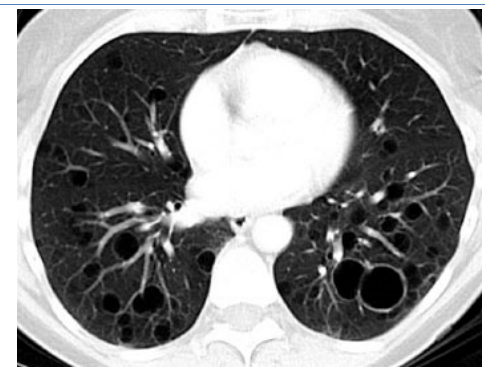

(a): Original CT-Lung image

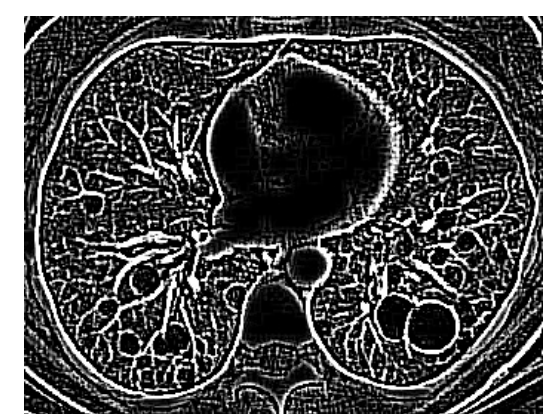

Result of the proposed $5 \times 5$ mask in $y$-direction

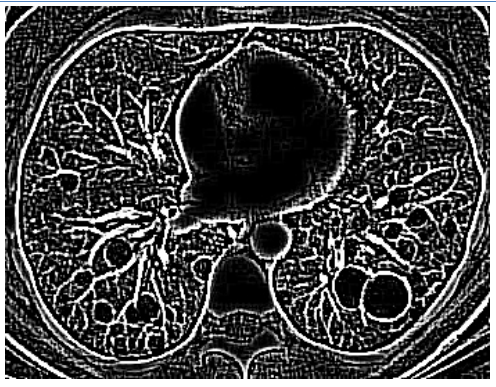

(b): Result of the proposed $5 \times 5$ mask in $\mathrm{x}$-direction

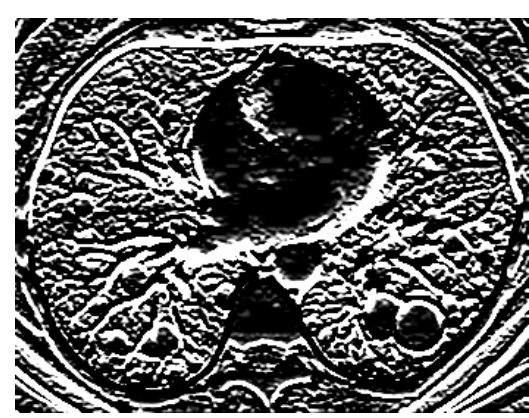

(d Sobel $5 \times 5$ Mask in x-direction

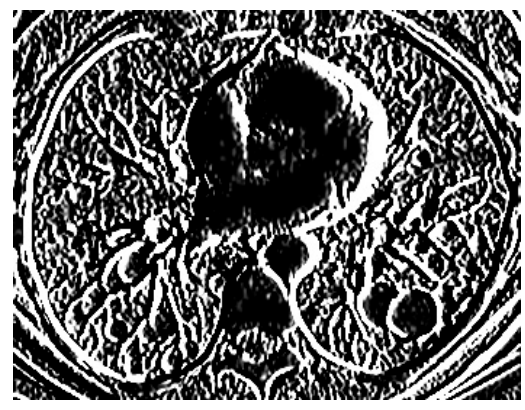

(e) : Sobel $5 \times 5$ Mask in y-direction

Figure 2. Results of our proposed method and 5x5 Sobel masks 


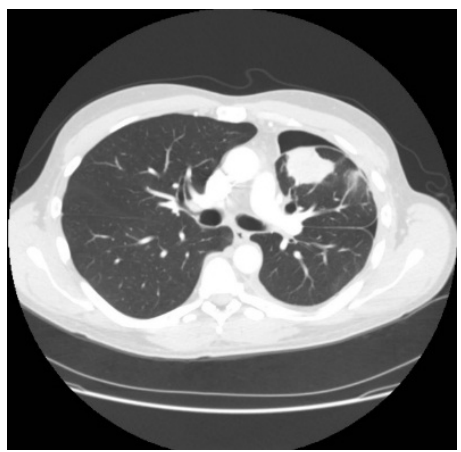

(a): Original CT-Lung image

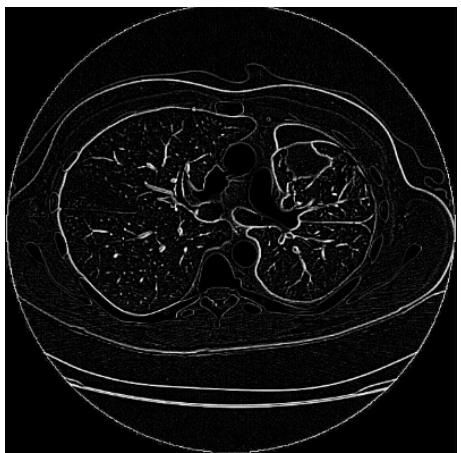

(c) Result of the proposed $5 \times 5$ mask in $y$-direction

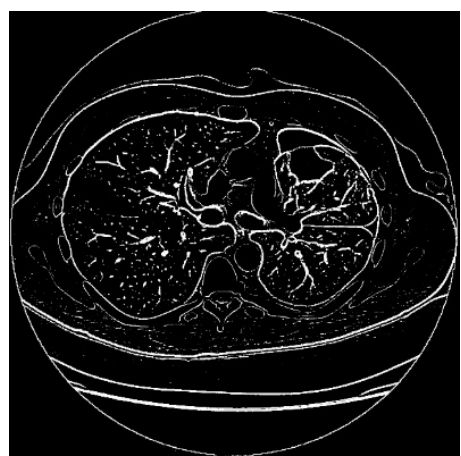

(b): Result of the proposed $5 \times 5$ mask in $\mathrm{x}$ direction

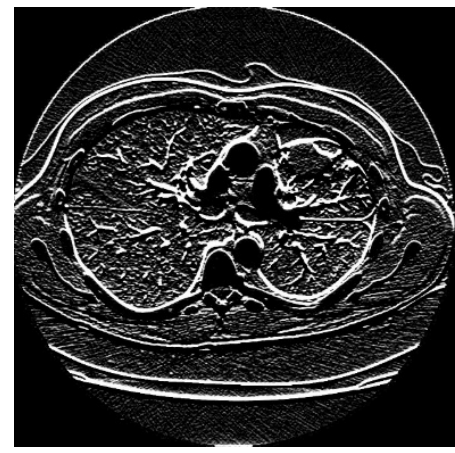

(d) Sobel $5 \times 5$ Mask in x-direction

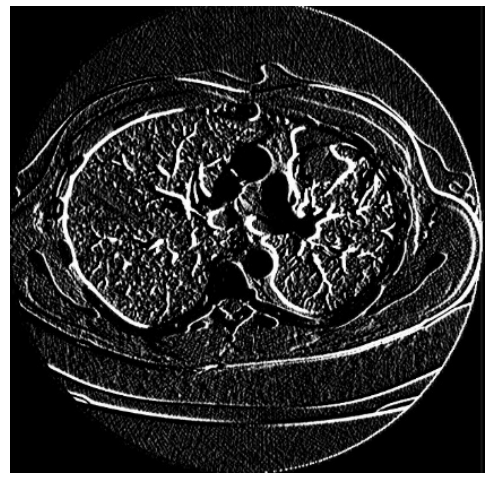

(e) Sobel $5 \times 5$ Mask in y-direction

Figure 3. Results of our proposed method and 5x5 Sobel masks

\section{Conclusion and Future Works}

In this paper, we presented a new method for detecting edges in CT-Scan lung images. The proposed method is based on the finite difference method. In previous operator [15], the masks are constructor using 2 parameters but in this paper we added a third parameter in order to have more general masks. 
Ali El-Zaart and Toufic El-Arwadi; A New Edge Detection Method for CT-Scan Lung Images. Journal of Biomedical Engineering and Medical Imaging, Volume 2, No 5, October (2015) , pp 1-9

The new masks are applied three CT-Scan images and we obtained good results in comparison with Sobel $5 \times 5$ masks. The objective of the proposed method is to detect edges in lung images, as future work, we have to develop another method in order to reconstruct the entire boundaries of lung in order to define well the candidate cancer region.

\section{REFERENCES}

[1] Gonzalez R. and Woods R., "Digital image processing," 3rd Edition, Prentice Hall, New York, 2008, pp. 695.

[2] Edge Detection and Segmentation of Multiple Contours from CT Scan Images Amba D. Bhatt, Ujjaval Gupta , Vishal Wagholikar, Uday V. Pise Computer-Aided Design and Applications Vol. 9, Iss. 4, 2012

[3] COMPARATIVE ANALYSIS OF VARIOUS IMAGE EDGE DETECTION TECHNIQUES FOR TWO DIMENSIONAL CT SCAN NECK DISC IMAGE, J Preetha, S Selvarajan and P Suresh, International Journal of Computer Science \& Communication, Vol. 3, No. 1, January-June 2012, pp. 57-61.

[4] EDGE DETECTION FROM CT IMAGES OF LUNG , S. K. BANDYOPADHYAY, INTERNATIONAL JOURNAL OF ENGINEERING SCIENCE \& ADVANCED TECHNOLOGY, Volume - 2, Issue - 1, $34-37$.

[5] A Novel Approach of Cancerous Cells Detection from Lungs CT Scan Images, N. Pandey ,S. Nandy International Journal of Advanced Research in Computer Science and Software Engineering, Volume 2, Issue 8, August 2012.

[6] T. El-Arwadi and A. El-Zaart, A Novel 5x5 Edge Detection Operator For Blood Vessel Images, accepted at the British Journal of Applied Science \& Technology, accepted August 2015.

[7] Wafaa Kamel Al-Jibory and Ali El-Zaart "A New Edge Detector in CT Scan Images Using Gradient of Weibull Distribution", World of Computer Science and Information Technology Journal (WCSIT), Vol. 4, No. 1, 13-17, 2014.

[8] Canny J. "A Computational Approach to Edge Detection Edge Detection". IEEE Transactions on Pattern Analysis and machine intelligence, Vol. PAMI-8, NO. 6, November 1986. 